\title{
Osteomalacia: desde la sospecha clínica al tratamiento. Revisión del tema
}

\section{Osteomalacia: from clinical suspicion to treatment: topic review}

\author{
Duque $V^{1}$, Barrera $N^{1}$, Gómez $J^{1}$, Guerra $V^{1}$, Ceballos L ${ }^{1}$, Aristizábal $N^{2}$.
}

${ }^{1}$ Estudiante pregrado de Medicina, Universidad Pontificia Bolivariana, Facultad de Medicina. Medellín, Colombia.

${ }^{2}$ Médico internista-Endocrinólogo, Clínica Universitaria Bolivariana, Clínica Las Américas, Medellín, Colombia.

Autor de correspondencia: Valentina Duque Cano

Correo electrónico: vduquecano29@gmail.com

Fecha de recepción: 01/04/2019

Fecha de aceptación: 29/08/2019

\section{Resumen}

El propósito de este artículo es presentar una revisión de la literatura acerca de la epidemiología, fisiopatología, manifestaciones clínicas y manejo de la osteomalacia. De este modo, se busca brindar información actualizada que conduzca a una mejor comprensión de la enfermedad y un adecuado tratamiento. Para una correcta mineralización ósea es necesario mantener un aporte adecuado de vitamina D y que los distintos procesos metabólicos que rigen sus efectos fisiológicos permanezcan indemnes, considerando que el papel de la vitamina $\mathrm{D}$ en el organismo es mantener la homeostasis del calcio y la salud ósea. El déficit de esta vitamina puede producir raquitismo durante la infancia y osteomalacia en adultos. El aporte de vitamina $\mathrm{D}$ al organismo puede darse por dos vías: de manera endógena, mediante la exposición de la piel a la luz solar; o de manera exógena, a partir de la dieta. Cualquier condición que produzca una alteración de las dos vías y genere un déficit significativo de vitamina D o de sus efectos fisiológicos puede manifestarse en los adultos como osteomalacia. En Colombia la deficiencia de vitamina D tiene una prevalencia de alrededor del 55,3\%. Las manifestaciones clínicas de la osteomalacia, considerando la amplia variedad de signos y síntomas, pueden denominarse conjuntamente como síndrome osteomalácico. Dadas las múltiples causas que pueden devenir en la osteomalacia, lo más adecuado es identificar la etiología que da origen al cuadro clínico para establecer un esquema terapéutico efectivo.

Palabras clave: osteomalacia, deficiencia de vitamina D, hipofosfatemia, hipocalcemia, calcificación fisiológica.

\section{Abstract}

This review it's proposed to explore updated bibliography taking in a count the epidemiology, physiopathology, clinical manifestations and treatment of Osteomalacia to achieve a better comprehension about the disease and a proper specific treatment to this condition. It's known that for a correct mineralization it's necessary an appropriate input of vitamin $D$ and it's metabolic processes must remain unscathed. Vitamin $D$ role in the human body is to maintain the homeostatic balance of calcium and the bone health; vitamin D deficit can cause Rickets in childhood and Osteomalacia in adults; this way, the most frequent cause of Osteomalacia it's the vitamin D deficiency and in Colombia it's prevalence is around 55,3\%. Vitamin D is provided to the organism through two ways: endogenously from the solar exposition of the skin or exogenously from the diet. Any condition that alterate one of the two ways and produce a significant deficit of vitamin D or a detriment of its physiological effects can manifestate as osteomalacia. The clinical manifestations of osteomalacia, considering the wide variety of signs and symptoms, can be referred to jointly as Osteomalacia Syndrome. On the grounds that a multiple causes can produce osteomalacia, it's advisable to identify the etiology of the clinical manifestations to establish an effective therapeutic scheme.

Keywords: osteomalacia, vitamin $D$ deficiency, hypophosphatemia, hypocalcemia, physiologic calcification.

\section{Introducción}

La osteomalacia es un defecto o retraso de la mineralización de la matriz orgánica del hueso u osteoide ${ }^{(1)}$ en sitios de recambio óseo ${ }^{(2)}$. Este término se suele confundir con otras entidades como el raquitismo y la osteoporosis. Es una alte- 
ración clínica diferente al raquitismo, el cual hace referencia a la deficiencia de mineralización, específicamente en el cartílago de crecimiento, por lo que, a diferencia de la osteomalacia, no es posible que se produzca en adultos después del cierre epifisario $^{(1)}$. Por otro lado, se distingue de la osteoporosis, ya que esta última se produce por una reabsorción excesiva del hueso existente; y en la osteomalacia el defecto radica sobre la formación de hueso nuevo ${ }^{(2)}$.

\section{Métodos}

Se realizó una revisión de tema. Las palabras clave utilizadas para la búsqueda fueron: osteomalacia, vitamin $D$ deficiency, hypophosphatemia, hypocalcemia, physiologic calcification, correspondientes a su traducción en español con base en el Medical Subject Heading $(\mathrm{MeSH})^{(3)}$.

\section{Definición}

La osteomalacia es un problema de la mineralización del osteoide que recién se forma en los lugares de recambio óseo, el cual constituye, por lo tanto, un trastorno en el proceso de remodelado óseo del hueso maduro ${ }^{(1,2)}$.

\section{Epidemiología}

La prevalencia e incidencia de la osteomalacia es difícil de precisar, debido a que es una enfermedad comúnmente asintomática; la mayoría de las veces se encuentra asociada con osteoporosis en ancianos ${ }^{(4)}$ y con su etiología multifactorial ${ }^{(5)}$. Se estima que la prevalencia descrita en pacientes ancianos va del $1 \%$ (determinada en autopsias) hasta el $18 \%{ }^{(4)}$. La causa más frecuente de osteomalacia es la deficiencia de vitamina $\mathrm{D}^{(6)}$, la cual es una condición frecuente. En un estudio realizado en Estados Unidos se encontró que la prevalencia de deficiencia de vitamina D era del 41,6\%, siendo más frecuente en raza negra e hispanos ${ }^{(7)}$. En la actualidad, no hay estudios en los que se determine la prevalencia de osteomalacia en Colombia.

\section{Metabolismo de la vitamina D}

\section{Síntesis de $1,25(\mathrm{OH})_{2} \mathrm{D}_{3}$ a partir de la vitamina $\mathrm{D}_{3}$}

La vitamina $\mathrm{D}_{3}$ (colecalciferol) puede ser sintetizada en la piel a partir de 7-dehidrocolesterol, mediante un desdoblamiento fotoquímico en respuesta a la radiación ultravioleta, o también puede provenir de la dieta: productos lácteos, aceites de pescado (arenque, bacalao) y las yemas de huevo ${ }^{(8-10)}$. La vitamina D producida a partir del 7-dehidrocolesterol depende de la intensidad de la exposición a la radiación ultraviole$\operatorname{ta}^{(11)}$. La vitamina $\mathrm{D}$ de origen vegetal se encuentra en forma de vitamina $D_{2}$, mientras que la procedente de animales está en forma de vitamina $D_{3}$. Estas dos formas tienen una actividad biológica equivalente y se activan con la misma eficacia, por medio de las hidroxilasas de vitamina $\mathrm{D}$ en el ser humano ${ }^{(12)}$.

Para que la vitamina D pueda ejercer sus efectos fisiológicos debe ser convertida a su forma activa. Desde su absorción en el intestino o su producción en la piel, la vitamina D viaja por el torrente sanguíneo unida a la proteína transportadora de vitamina D (DBP, por sus siglas inglés: vitamin $D$ binding protein), que se encarga de llevarla al hígado y al riñón.

En el hígado, la vitamina $\mathrm{D}_{3}$ sufre una 25-hidroxilación por enzimas semejantes a las del citocromo P-450, cuyo producto es la formación de 25-hidroxivitamina $\mathrm{D}_{3}$ o $25(\mathrm{OH}) \mathrm{D}_{3}$. Esta enzima (la 25-hidroxilasa) no se encuentra muy bien regulada y, por ende, la $25(\mathrm{OH}) \mathrm{D}_{3}$ es la principal forma circulante y de depósito de la vitamina $\mathrm{D}^{(6)}$. Luego, la $25(\mathrm{OH}) \mathrm{D}_{3}$ es llevada por la DBP al riñón, donde es nuevamente hidroxilada por la enzima 25(OH)D-1 $\alpha$-hidroxilasa, que está presente en las células del túbulo contorneado proximal.

El resultado final es la 1,25-dihidroxivitamina D o $1,25(\mathrm{OH})_{2} \mathrm{D}_{3}$ (calcitriol) hormonalmente activa, que es la responsable de la mayoría de los efectos fisiológicos de la vitamina D. La hormona paratiroidea (PTH) estimula la hidroxilasa renal, en tanto que el calcio $\left(\mathrm{Ca}^{2+}\right)$ y el factor de crecimiento fibroblástico 23 (FGF23) -que es secretado por los osteocitos y que funciona en el riñón como una hormona fosfatúrica; y en el intestino, inhibiendo la absorción de fosfato- la reprimen. La $1 \alpha$-hidroxilasa también es inhibida por el producto de la acción de la enzima, la $1,25(\mathrm{OH})_{2} \mathrm{D}_{3}{ }^{(11)}$.

La principal vía de inactivación de los metabolitos de la vitamina $\mathrm{D}$ es un paso de hidroxilación adicional que realiza vitamina D 24-hidroxilasa (CYP24R1), una enzima que se expresa en casi todos los tejidos. La 24-hidroxilasa puede hidroxilar tanto la $25(\mathrm{OH}) \mathrm{D}_{3}$ como la $1,25(\mathrm{OH})_{2} \mathrm{D}_{3}$. Sin embargo, se ha sugerido que esta última es el principal inductor de la vitamina D 24-hidroxilasa y promueve así su propia inactivación. Finalmente, la 24-hidroxilasa facilita la conversión de la $1,25(\mathrm{OH})_{2} \mathrm{D}_{3}$ a 1,24,25(OH) ${ }_{3} \mathrm{D}_{3}$ y luego a ácido calcitroico (metabólicamente inactivo), que es excretado en la bilis ${ }^{(9,11,12)}$ (Figura 1).

\section{Efectos fisiológicos de la $1,25(\mathrm{OH})_{2} \mathrm{D}_{3}$ o calcitriol}

La $1,25(\mathrm{OH})_{2} \mathrm{D}_{3}$ ejerce sus efectos por medio de su unión a un receptor nuclear denominado receptor de vitamina $D$ (VDR, por sus siglas en inglés: vitamin $D$ receptor), que está presente en el sistema musculoesquelético (osteoblastos, condrocitos y músculo estriado esquelético), gastrointestinal y hepático, endocrino (paratiroides, células $C$ tiroideas, células $\beta$ pancreáticas, glándulas suprarrenales e hipófisis), renal (células tubulares, aparato yuxtaglomerular y podocitos), entre otros ${ }^{(13)}$. La $1,25\left(\mathrm{OH}_{2}\right) \mathrm{D}_{3}$ actúa como un mensajero químico que induce respuestas genómicas y no genómicas al unirse a su receptor ${ }^{(14)}$. 
Figura 1. Metabolismo de la vitamina D.

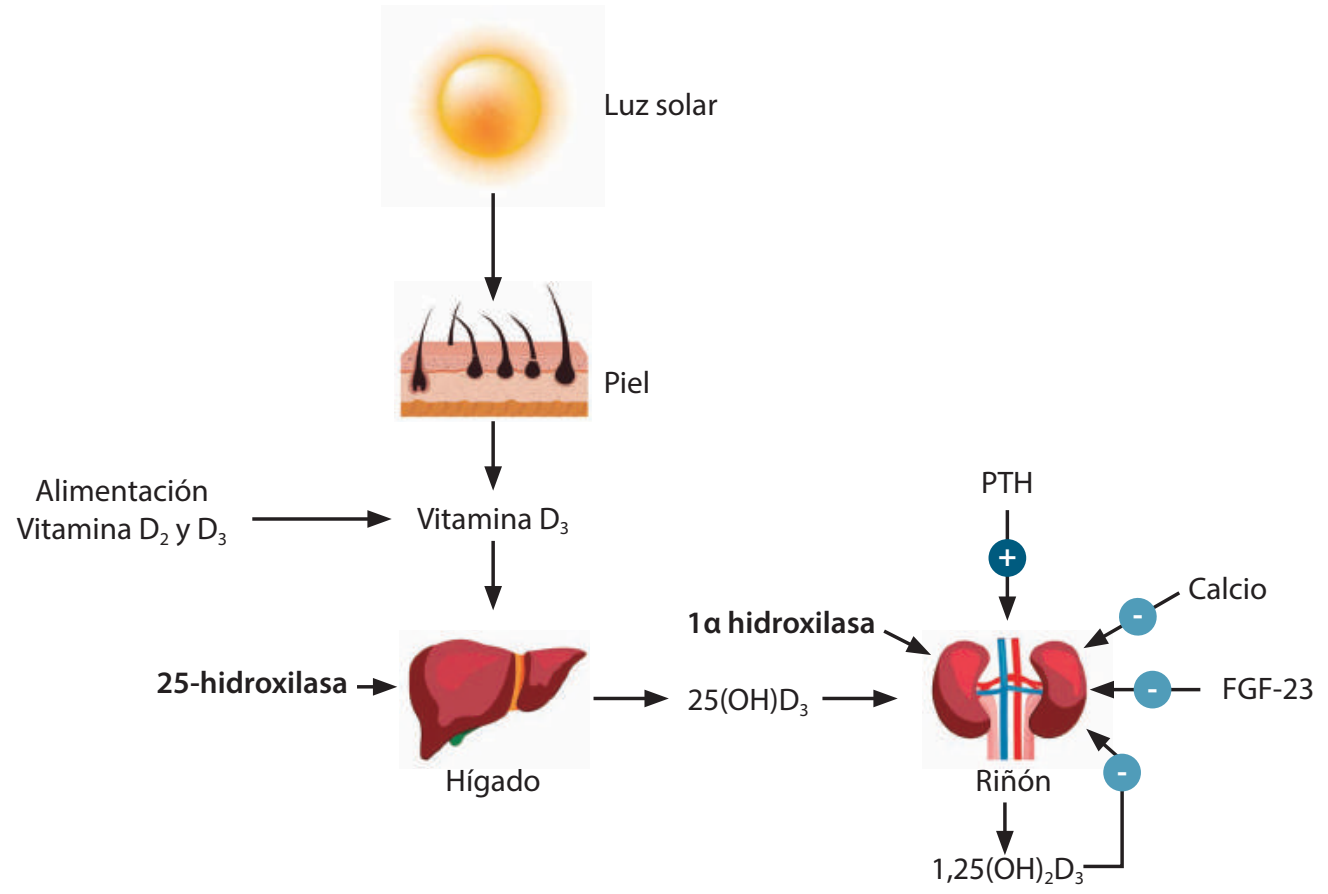

La $1,25(\mathrm{OH})_{2} \mathrm{D}_{3}$ es esencial para la interacción entre los riñones, huesos, glándula paratiroides e intestino, manteniendo los niveles de calcio dentro de unos límites estrechos ${ }^{(13)}$. Además, conserva las concentraciones séricas de calcio y fosfato, para mantener la mineralización de la matriz ósea previamente no mineralizada ${ }^{(13)}$.

\section{Fisiopatología de la osteomalacia}

Las consecuencias de la deficiencia de vitamina D sobre la salud ósea incluyen: osteomalacia y raquitismo ${ }^{(10)}$, ambas relacionadas con un defecto en la mineralización ósea. En el proceso de remodelado óseo fisiológico en el adulto, durante la fase de formación ósea, los osteoblastos sintetizan colágeno y otras proteínas de la matriz ósea, como constituyentes de una sustancia denominada osteoide. En una fase subsiguiente se produce la mineralización de este osteoide con el depósito de calcio y fósforo, para formar los cristales hidroxiapatita del tejido óseo maduro sin la participación directa de los osteoblastos. Los minerales óseos son depositados inicialmente como fosfato cálcico amorfo y, luego, se convierten a la forma cristalina de la hidroxiapatita. El calcio requerido para este proceso es tomado del espacio extracelular y transportado a las vesículas de la matriz ${ }^{(6)}$.

Para que el proceso de mineralización ósea se lleve a cabo correctamente, el hueso depende directamente de la suficiencia de calcio y fósforo, de la presencia de una matriz de colágeno normal, de la ausencia de inhibidores de la mineralización (como el pirofosfato) y de una cantidad adecuada de fosfatasa alcalina ósea (una enzima que proviene de la actividad osteoblástica y que es imprescindible para la disponibilidad de fosfato y una correcta mineralización de la matriz; tal efecto lo consigue al hidrolizar el pirofosfato). Los defectos en estos requisitos constituyen la causa de la mayoría de las formas de osteomalacia $^{(1,6)}$.

Osteomalacia significa reblandecimiento del hueso. Hasta el $70 \%$ de la mineralización del osteoide depositado por los osteoblastos avanza aproximadamente $1 \mu \mathrm{m}$ por día; no obstante, en la osteomalacia la mineralización se enlentece o detiene completamente, mientras que los osteoblastos continúan produciendo osteoide, que se acumula en cantidades excesivas. En último término, la acumulación de un osteoide no mineralizado tiene como producto resultante un hueso

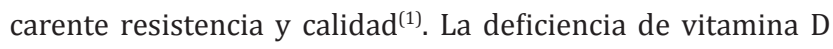
prolongada en el tiempo puede conducir a hipocalcemia con hiperparatiroidismo secundario, hipofosfatemia y, finalmente, osteomalacia $^{(6)}$

\section{Etiología de la osteomalacia}

A pesar de que es una entidad poco frecuente, las posibles causas de osteomalacia son muy numerosas y pueden clasificarse dentro de tres grupos ${ }^{(6,8,15)}$ (Tabla $\mathbf{1}$ ).

- Alteraciones en el metabolismo de la vitamina D

- Alteraciones en el metabolismo del fósforo

- Acumulación de inhibidores de la mineralización. 
Tabla 1. Causas más frecuentes de osteomalacia

\section{Osteomalacia por alteraciones en el metabolismo de la vitamina D}

Malabsorción de la vitamina D

Falta de exposición solar o de aporte exógeno de vitamina $D$

Síndrome nefrótico

Aumento del catabolismo de la vitamina D

Alteración de la hidroxilación hepática o renal

Osteomalacia hipofosfatémica

Disminución de la reabsorción tubular de fosfatos

$\checkmark$ Congénitas: raquitismos hipofosfatémicos

$\checkmark$ Adquiridas

- Osteomalacia tumoral

- Disfunción del túbulo renal (síndrome de Fanconi y acidosis tubular renal)

Inhibidores de la mineralización

Acidosis crónica

Intoxicación por aluminio

Flúor

Etidronato

Ingesta de calcio deficiente (niños)

Osteomalacia axial

Fibrogénesis imperfecta

Tomada y modificada de la referencia 6 .

\section{Alteraciones en el metabolismo de la vitamina D}

La deficiencia de vitamina $\mathrm{D}$ es la causa más frecuente de osteomalacia. Los trastornos del aparato digestivo, hepáticos o pancreáticos pueden ser el origen de los problemas de la vitamina D. La gastrectomía y la cirugía para el tratamiento de la obesidad son algunas de estas causas. En las enfermedades que cursan con malabsorción, como enteropatías inflamatorias, enfermedad celíaca o pancreatitis crónica, el problema no suele ser tanto por el déficit de absorción de la vitamina $\mathrm{D}$ de la dieta, sino por la interrupción de la circulación enterohepática de metabolitos de la vitamina D. En ese sentido, también juegan un papel importante la hepatopatía o la nefropatía, que se caracterizan por defectos en la 25-hidroxilación o en la $1 \alpha$-hidroxilación, respectivamente, así como síndromes nefróticos en los cuales la pérdida de vitamina D ligada a proteínas es considerable ${ }^{(6)}$.

Además, existen dos trastornos hereditarios que se acompañan de un pseudodéficit de vitamina $\mathrm{D}$, los denominados raquitismos vitamino-D-dependientes (VDDR) tipo I y II. El VDDR-I se debe a una ausencia de la $1 \alpha$-hidroxilasa renal por mutación del gen que la codifica, mientras que el VDDR-II es una alteración genética del receptor de la vitamina $\mathrm{D}^{(6)}$.

\section{Alteraciones en el metabolismo del fósforo}

En este grupo de osteomalacias se incluyen una serie de trastornos en los que hay una pérdida anormal de fosfato por el riñón e hipofosfatemia. En la homeostasis humoral del fosfato participan el calcitriol y la PTH, así como el FGF-23; y pueden clasificarse como hereditarias o adquiridas ${ }^{(6)}$

\section{Causas hereditarias}

- Raquitismo hipofosfatémico ligado al cromosoma X: es el más común y supone $80 \%$ de los casos. Se debe a una mutación en el gen phosphate regulating gene with homologies to endopeptidases on the X chromosome (PHEX, por sus siglas en inglés). Se da por diversos mecanismos, entre ellos la elevación del FGF-23, que causa un incremento de la fosfaturia y, como consecuencia, hipofosfatemia.

- Raquitismo hipofosfatémico autosómico: está relacionado con mutaciones del gen que codifica el FGF-23 situado en el cromosoma 12, que da como resultado un FGF-23 más resistente a la degradación por proteinasas.

- Raquitismo hipofosfatémico con hipercalciuria: es muy raro. El trastorno genético responsable está probablemente relacionado con defectos en las proteínas implicadas en la reabsorción tubular renal del fósforo. Puesto que cursa con un aumento de los niveles séricos de $1,25(\mathrm{OH})_{2} \mathrm{D}_{3}$, se manifiesta con hipercalciuria ${ }^{(6)}$.

\section{Causas adquiridas}

- Osteomalacia tumoral: está asociada con tumores benignos de tamaño pequeño y de origen mesenquimatoso, difíciles de localizar. Aunque también se ha descrito que se asocia con carcinoma de mama, próstata y pulmón. El tumor produce FGF-23, por lo que se disminuye la reabsorción tubular de fosfatos y la hidroxilación renal de la vitamina $\mathrm{D}^{(6)}$.

- Disfunción del túbulo renal: se da por el síndrome de Fanconi y la acidosis tubular renal, que puede ser multicausal $^{(6)}$.

\section{Manifestaciones clínicas}

El hueso poco mineralizado tiene menor resistencia, es más maleable y está predispuesto a las fracturas. Con frecuencia esta situación es asintomática; sin embargo, los pacientes pueden tener manifestaciones relacionadas con la debilidad ósea y las alteraciones del metabolismo fosfocálcico ${ }^{(16)}$. En su forma clásica, la osteomalacia se caracteriza por tener múltiples signos y síntomas que pueden denominarse colectivamente síndrome osteomalácico. Algunos síntomas son vagos e inespecíficos; no obstante, hay otros síntomas que son altamente específicos y, a menudo, diagnósticos; es necesario un alto grado de sospecha en el contexto clínico adecuado, especialmente en las etapas iniciales ${ }^{(6,17)}$. 
El dolor en la osteomalacia es sordo y poco localizado, pero se siente claramente en los huesos. El dolor óseo suele ser persistente y empeora por el peso o la contracción de los músculos durante la locomoción ${ }^{(16)}$. Rara vez se alivia completamente con el descanso, suele ser simétrico y difuso, que comienza en la parte inferior de la espalda, luego se extiende a la cintura pélvica, las caderas y la parte superior de los muslos y las costillas. La compresión lateral de las costillas o de la cintura pélvica, y la compresión del esternón son maniobras clínicas útiles para provocar dolor en casos leves a moderados ${ }^{(6,17)}$.

La debilidad muscular puede variar desde una anomalía sutil detectable solo con un examen físico cuidadoso, hasta una discapacidad grave que puede llegar a provocar una parálisis completa. Los síntomas específicos incluyen dificultad para levantarse de una silla, subir o bajar escaleras, y el modo de caminar característico causado por la incapacidad para levantar la pierna del suelo, debido a la debilidad del cuádriceps. La marcha anormal puede ser el resultado de dolor o debilidad ${ }^{(6)}$.

En pacientes con formas genéticas de osteomalacia, se pueden observar deformidades residuales de raquitismo con estatura baja asociada. Las deformidades relacionadas con el ablandamiento del esqueleto adulto incluyen cifosis, genu varo, genu valgo, pectus carinatum, protrusio acetabuli y pelvis trirradiada con un arco pubiano estrecho (Figura 2), que son poco comunes en la práctica contemporánea ${ }^{(1,6,18-21)}$.
Cuando existe hipocalcemia, pueden aparecer manifestaciones de tetania como parestesias, contracturas tónicas, convulsiones y signos de Chvostek y Trousseau. En las formas graves, pueden desarrollarse deformidades en el tórax (tórax en campana) y esternón (esternón en quilla) ${ }^{(16,22)}$.

Los múltiples cambios óseos que se presentan en esta patología se pueden detectar en los estudios radiográficos y básicamente son el resultado de un incremento en la secreción de PTH o de una alteración en la mineralización de la matriz ósea. El hiperparatiroidismo secundario, una consecuencia inevitable del agotamiento de la vitamina $\mathrm{D}$, conduce al adelgazamiento del hueso cortical. El aumento de la porosidad cortical se manifiesta como estrías corticales en los metacarpianos y las falanges. La manifestación radiográfica más común es la osteopenia. Se pueden ver cambios en la forma del hueso, como el protrusio acetabuli (Figura 2E), aunque son poco frecuentes en la actualidad.

Otro hallazgo muy característico de la osteomalacia, aunque igualmente infrecuente, son las fracturas por estrés (zona de Looser); estas son fisuras incompletas sin desplazamiento, que aparecen como resultado de traumas repetitivos. Las zonas afectadas con mayor frecuencia son las costillas, pubis, escápula, cara inferior del cuello femoral, cara medial del fémur proximal y los metatarsianos ${ }^{(16)}$.

Figura 2. Signos clínicos de la osteomalacia. A. Cifosis, pectus carinatum. B y C. Genu varum. D. Genu valgum. E. Protrusio acetabuli. Tomadas de las referencias 18, 19, 20 y 21.
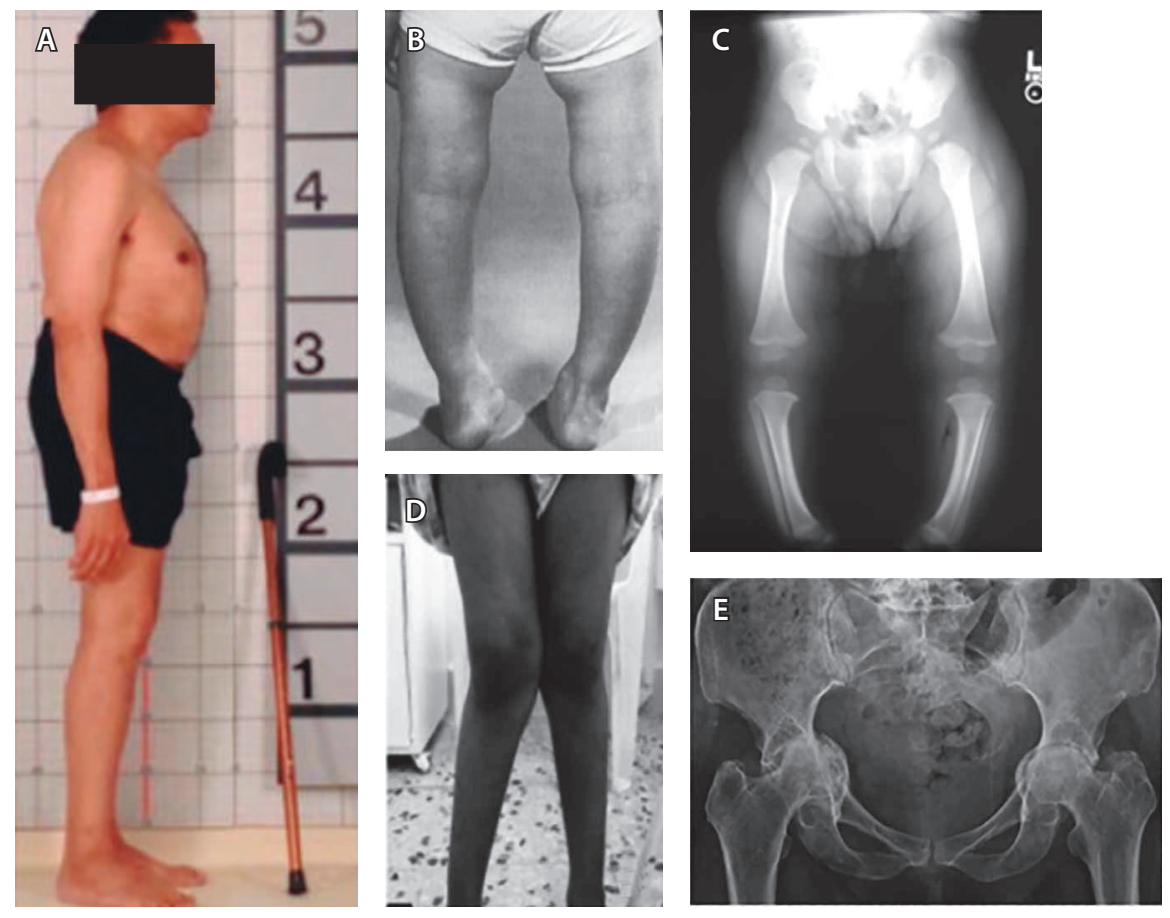


\section{Diagnóstico}

El diagnóstico se debe sospechar en los casos de dolor óseo en pacientes de alto riesgo. La densitometría ósea no se requiere para hacer el diagnóstico, dado que los hallazgos en estos casos son indistinguibles de los de la osteoporosis. El diagnóstico se basa en la combinación de los síntomas, signos clínicos y los hallazgos de laboratorios: calcio, fósforo, albúmina, 25(OH) vitamina D y hormona paratiroidea intacta; pocas veces se cuenta con la confirmación histológica de la enfermedad.

\section{Estudios de laboratorio}

Las anormalidades en las pruebas de laboratorio dependen directamente de la causa de la osteomalacia; las principales (evidenciadas en los casos de deficiencia de vitamina D) son ${ }^{(16)}$ :

- Niveles de 25(OH) vitamina D <10 -15 ng/mL (100 \%)

- Hormona paratiroidea elevada (100\%)

- Fosfatasa alcalina elevada (95 \%-100 \%)

- Calcio urinario bajo (87\%)

- Hipocalcemia e hipofosfatemia (25 \%-38 \%).

Al ser el déficit de vitamina D la principal causa de osteomalacia, se debe tener en cuenta que los niveles séricos de 25-hidroxivitamina D son el mejor indicador para conocer el estado general de la vitamina D, porque su medición refleja la vitamina D total ${ }^{(12)}$.

\section{Estudios radiográficos}

Están indicados en casos de dolor severo.

\section{Biopsia ósea}

Está indicada cuando el diagnóstico es dudoso; no está ampliamente disponible en nuestro medio.

\section{Tratamiento}

Se debe identificar la etiología. El tratamiento del déficit de vitamina D se basa en la observación de que 800 UI de esta vitamina con complementos de calcio disminuyen el riesgo de fracturas de la cadera en ancianos; por lo tanto, se considera que esta dosis constituirá el consumo diario adecuado para evitar la deficiencia en los adultos. En individuos que tienen disminución de la $1 \alpha$-hidroxilación, el tratamiento indicado incluye los metabolitos activos, como son $1,25(\mathrm{OH})_{2} \mathrm{D}_{3}$ (calcitriol 0,25 a 0,5 $\mu \mathrm{g}$ /

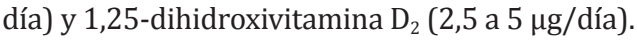

Si la vía necesaria para la activación de la vitamina se encuentra intacta, se puede tratar la deficiencia profunda (valores $<10 \mathrm{ng} / \mathrm{mL}$ ) con la repleción farmacológica; en la etapa inicial 50000 UI por semana, por tres a 12 semanas, seguida de dosis de sostenimiento de $800 \mathrm{UI} /$ día $^{(23)}$. Pueden ser necesarias dosis farmacológicas para el tratamiento de sostenimiento en pacientes que reciben medicamentos que aceleran el metabolismo del $1,25(\mathrm{OH})_{2} \mathrm{D}_{3}$ o producen resistencia. En casos especiales, como la cirugía bariátrica o en síndromes de malabsorción, se pueden requerir hasta 200000 UI semanales. La suplementación de calcio incluye la administración de 1,5 a 2,0 g de calcio elemental al día ${ }^{(23)}$.

Para hacer una vigilancia seriada y determinar la resolución de la deficiencia vitamínica, los métodos más eficaces incluyen las mediciones del calcio en suero y orina. En individuos en quienes se produjo la reposición de la vitamina y reciben complementos adecuados de calcio, la excreción de calcio en orina de 24 horas debe estar en 100 a 250-300 mg/24 horas. Se debe tener en cuenta que las concentraciones menores sugieren falta de adherencia al régimen terapéutico o una menor absorción de los suplementos de calcio o vitamina D. Las concentraciones mayores de $250 \mathrm{mg} / 24$ horas predisponen a nefrolitiasis, por lo que es necesario disminuir las dosis de la vitamina, del calcio o ambos ${ }^{(12,23)}$. El tratamiento se mantendrá hasta la resolución de la osteomalacia. La elevación de la calciuria y de la densidad mineral ósea indican que se ha resuelto el trastorno metabólico óseo, lo que se puede tardar muchos meses ${ }^{(6)}$.

La corrección de la hipofosfatemia debe estar guiada por la presencia de signos o síntomas, y el estado clínico general del paciente. También hay que tener en cuenta la presencia de insuficiencia renal, la administración concomitante de glucosa intravenosa y la posibilidad de empeorar la hipocalcemia coexistente. Hay pocos datos de estudios clínicos para calcular la dosis apropiada y la velocidad de administración de fosfato. En pacientes con niveles de fósforo $<2,5 \mathrm{mg} / \mathrm{dL}$, sin insuficiencia renal grave (etapas 1, 2 y 3) o hipocalcemia, la administración de 2 a $8 \mathrm{mmol} / \mathrm{h}$ de fósforo elemental intravenoso durante $4 \mathrm{a}$ 8 horas corrige frecuentemente la hipofosfatemia sin provocar hiperfosfatemia ni hipocalcemia ${ }^{(24)}$.

Es fundamental el seguimiento del calcio y del fosfato séricos cada 6 a 12 horas. La hipofosfatemia menos grave (valores mayores a $2,5 \mathrm{mg} / \mathrm{dL}$ ) se debe tratar con suplementos orales de fosfato si es posible, por lo general, en una dosis de 1 a $2 \mathrm{~g} /$ día (de fosfato elemental) de fosfato potásico o sódico neutro repartida tres o cuatro veces al día. Sin embargo, en muchos pacientes la administración oral de fosfato está limitada por náuseas o diarrea ${ }^{(24)}$.

El KRN23 (burosumab) es un anticuerpo monoclonal de inmunoglobulina $\mathrm{G}(\operatorname{IgG}) 1$ humano recombinante, que se une al FGF23 y bloquea su actividad. Tiene el potencial de mejorar los resultados bioquímicos y esqueléticos en adultos y niños con hipofosfatemia ligada al cromosoma $\mathrm{X}$, la cual se caracteriza por tener altos niveles del FGF23 debido a una mutación del gen PHEX ${ }^{(25-28)}$. En un estudio fase I, realizado en pacientes asiáticos, se demostró que el burosumab aumenta los niveles séricos de $1,25(\mathrm{OH})_{2} \mathrm{D}_{3}$, al neutralizar el efecto del FGF23 y mejora la hipofosfatemia. No hubo alteración de los niveles de calcio y paratohormona, no hubo efectos adversos signifi- 
cativos y se demostró, además, que no hay variaciones de su efectividad en las diferentes razas ${ }^{(29)}$.

Otro estudio realizado en pacientes americanos encontró que esta molécula mejora la capacidad funcional de estos pacientes, reduce síntomas como el dolor y la rigidez osteomuscular, y favorece la regeneración ósea en fracturas. Además, demostró un buen perfil de tolerabilidad en pacientes adultos y pediátricos; y los efectos adversos reportados eran leves a moderados (cefalea, reacciones locales en el sitio inyectado, náuseas, vómito, fiebre, mialgias, dolor dental, abscesos dentales y vértigo). En cuanto a los efectos adversos graves, se encontró hiperfosfatemia en el $7 \%$ de los adultos estudiados, reacciones de hipersensibilidad tanto en niños como en adultos, empeoramiento de cuadros de síndrome de piernas inquietas preexistentes y aún sigue en estudio si la estenosis espinal puede empeorar con el uso de burosumab ${ }^{(30)}$.

El tratamiento de la osteomalacia en el embarazo se basa en los reportes de casos, en los que las mujeres embarazadas con osteomalacia (diagnosticada en el momento del parto) fueron tratadas exitosamente con altas dosis de vitamina D (600 000 UI por vía intramuscular como dosis única después del parto) y suplementos de calcio (hasta 1,5 g por día). Sin embargo, la administración durante el embarazo de una dosis tan alta de vitamina $\mathrm{D}$ no se ha estudiado adecuadamente ${ }^{(31)}$.

En series de casos de la década de 1960, las mujeres embarazadas con osteomalacia fueron tratadas de manera segura con calcio y 3000 a 6000 UI de vitamina D al día. Otros estudios evalúan la administración de esta vitamina en mujeres embarazadas con deficiencia de esta, algunas de las cuales tenían elevaciones en la PTH en suero, pero ninguna tenía deficiencia de vitamina D de suficiente gravedad y duración como para causar osteomalacia. En estos ensayos, la administración de suplementos de vitamina D a las 12 a 27 semanas de gestación con 400, 800, 2000 o 4000 UI diarias de $\mathrm{D}_{3}$ o 200000 UI como dosis oral única fue segura y logró aumentar las concentraciones séricas de vitamina $\mathrm{D}^{(31)}$.

Las mujeres que son diagnosticadas con osteomalacia durante el embarazo deben recibir calcio adecuado (apro- ximadamente 1000 a $1500 \mathrm{mg}$ diarios) y vitamina D. Típicamente, comienzan con 2000 a 4000 UI al día. Se debe hacer seguimiento midiendo la 25-hidroxivitamina D (25[OH] D) en suero, el calcio y la excreción urinaria de este después de un mes y tres meses; luego, con menos frecuencia (cada 6 a 12 meses), hasta que la excreción urinaria de calcio de 24 horas sea normal. Si con la dosis inicial la 25(OH) D sérica no mejora después de tres a cuatro meses de tratamiento, la dosis de vitamina D puede aumentar de 1000 a 2000 UI por día. La dosis de vitamina D se debe disminuir, según sea necesario, para prevenir la hipercalcemia o la hipercalciuria ${ }^{(31)}$.

El raquitismo hipofosfatémico hereditario se trata con una combinación de suplementos de fosfato y calcitriol. La osteomalacia inducida por un tumor se trata de manera similar, hasta que sea posible el tratamiento del tumor causante; o, indefinidamente, si esto no es posible. La osteomalacia por acidosis tubular renal se trata inicialmente con 5000 a 10000 UI por día de vitamina $\mathrm{D}$ y la acidosis se corrige con citrato de sodio y/o potasio. La hipofosfatasia es un trastorno raro del metabolismo con pocas opciones de tratamiento, se ha venido utilizando la terapia de reemplazo enzimático asfotasa alfa (Strensiq ${ }^{\circledR}$ ).

\section{Conclusiones}

La osteomalacia se caracteriza por una alteración en la mineralización de la matriz orgánica ósea en sitios de recambio, la causa más frecuente es la deficiencia de vitamina $\mathrm{D}_{3}$. Cursa con dolor y sensibilidad en los huesos, con la consecuente debilidad muscular y dificultad para caminar. Una sospecha clínica temprana en individuos con factores de riesgo, así como el diagnóstico y tratamiento oportuno permitirán impactar de forma positiva en la calidad de vida del paciente.

\section{Conflictos de interés}

Los autores declaran no hacer parte de actividades financieras relacionadas con otras empresas, ni estar involucrados en alguna situación que haga que la capacidad de juzgar sobre un asunto esté influenciada por algún interés.

\section{Referencias}

1. Weinstein R. Osteomalacia y raquitismo. En: Elsevier, editor: Goldman L, Schafer AI. Goldman-Cecil: tratado de medicina interna. 2017. p. 16451649.

2. Sud K, Parashar A, Chauhan V. Clinical Syndromes of Vitamin D and Phosphate Dysregulation. En: Williams R. Williams textbook of endocrinology. 13th edition. Melmed S, Polonsky KS, Larsen PR, Kronenberg H, editores. Philadelphia, PA: Elsevier; 2016. p. 373-388.

3. MeSH Browser [Internet]. [citado 7 de octubre de 2019]. Disponible en: https://meshb.nlm.nih.gov/search

4. Mazanec DJ, Khalaf T, Orr R. Metabolic bone disease. En: Benzel EC, Steinmetz MP. Benzel's spine surgery: techniques, complication avoidance, and management. 2017. p. 893-903.

5. Sundaram M, Schils J. Hyperparathyroidism, renal osteodystrophy, osteomalacia and rickets. En: Pope TL, Bloem HL, Beltran J, Morrison WB, Wilson DJ. Musculoskeletal imaging [Internet]. 2015 [citado 7 de octubre de 2019]. Disponible en: https://www.clinicalkey.com/dura/browse/ bookChapter/3-s2.0-C20100686676. p. 889-889.
6. Moreno AQ González MDS, Calleja $\mathrm{CH}$, Morales CM, Pino-Montes J del. Osteomalacia. Med - Programa Form Médica Contin Acreditado. 2016;12(16):909-14.

7. Forrest KYZ, Stuhldreher WL. Prevalence and correlates of vitamin D deficiency in US adults. Nutr Res. 2011;31(1):48-54.

8. Marazuela M. Déficit de vitamina D en el adulto: clínica, diagnóstico y tratamiento. Endocrinol Nutr. 2005;52(5):215-23.

9. Bringhurst FR, Demay MB, Krane SM, Kronenberg HM. Bone and mineral metabolism in health and disease. En: Kasper D, Fauci A, Hauser S, Longo D, Jameson JL, Loscalzo J, editores. Harrison's Principles of Internal Medicine [Internet]. 19. a ed. New York, NY: McGraw-Hill Education; 2015 [citado 5 de mayo de 2018]. Disponible en: accessmedicine.mhmedical.com/ content.aspx?aid=1120816741

10. Binkley N, Ramamurthy R, Krueger D. Low Vitamin D Status: Definition, Prevalence, Consequences, and Correction. Endocrinol Metab Clin North Am. 2010;39(2):287-301. 
11. Christakos S, Ajibade DV, Dhawan P, Fechner AJ, Mady LJ. Vitamin D: Metabolism. Endocrinol Metab Clin North Am. 2010;39(2):243-53.

12. Rosen CJ. Vitamin D Insufficiency. N Engl J Med. 2011;364(3):248-54.

13. Espinosa NAZ, Velásquez JMA, González VB, Blanco KEJ, Maya GC. Vitamina D: nuevos paradigmas. Med Lab. 2011;17(05-06):211-46.

14. Goltzman D. Functions of vitamin D in bone. Histochem Cell Biol. abril de 2018;149(4):305-12.

15. Manzano G, Vega MEA de la, Calleja CH, Morales CM, Montes J del P. Protocolo diagnóstico y tratamiento de la osteomalacia. Med Programa Form Médica Contin Acreditado. 2018;12(60):3542-6.

16. Bhan A, Rao AD, Rao DS. Osteomalacia as a result of vitamin D deficiency. Endocrinol Metab Clin North Am. junio de 2010;39(2):321-31, table of contents.

17. Walker J. Pathogenesis, diagnosis and management of osteomalacia. Nurs Older People. 2014;26(6):32-7.

18. Chong WH, Molinolo AA, Chen CC, Collins MT. Tumor-induced osteomalacia. Endocr Relat Cancer. 2011;18(3):R53-77

19. Rader CP, Corsten N, Rolf O. Osteomalacia and vitamin D deficiency. Orthopade. 2015;44(9):695-702.

20. Sahebari M, Sigari SY, Heidari H, Biglarian O. Osteomalacia can still be a point of attention to celiac disease. Clin Cases Miner Bone Metab. 2011;8(3):14-5.

21. Vaishya R. Bilateral protrusio acetabuli of the hip. BMJ Case Rep. 2014;2014. pii: bcr2013201784

22. Riancho J. Osteomalacia y raquitismo. REEMO. 2004;13(4):77-9.

23. Potts JT, Jüppner H. Trastornos de las glándulas paratiroides y la homeostasis de calcio. En: Kasper DL, Hauser SL, Jameson LJ, Fauci AS, Longo DL, Loscalzo J, editores. Harrison Principio de Medicina Interna (19.ㄹ edición). New York: Mc Graw Hill; 2015. p. 2466-88.
24. Bringhurst RF, Demay MB, Kronenberg HM. Hormonas y trastornos del metabolismo mineral. En: Elsevier, editor. Williams. Tratado de endocrinología (13.a edición). Barcelona: Elsevier; 2017. p. 1254-322.

25. Imel EA, Zhang X, Ruppe MD, Weber TJ, Klausner MA, Ito T, et al. Prolonged Correction of Serum Phosphorus in Adults With X-Linked Hypophosphatemia Using Monthly Doses of KRN23. J Clin Endocrinol Metab. 2015;100(7):2565-73.

26. Insogna KL, Briot K, Imel EA, Kamenický P, Ruppe MD, Portale AA, et al. A Randomized, Double-Blind, Placebo-Controlled, Phase 3 Trial Evaluating the Efficacy of Burosumab, an Anti-FGF23 Antibody, in Adults With XLinked Hypophosphatemia: Week 24 Primary Analysis. J Bone Miner Res Off J Am Soc Bone Miner Res. 2018;33(8):1383-93.

27. Lamb YN. Burosumab: First Global Approval. Drugs. 2018;78(6):707-14

28. Portale AA, Carpenter TO, Brandi ML, Briot K, Cheong HI, Cohen-Solal M, et al. Continued Beneficial Effects of Burosumab in Adults with X-Linked Hypophosphatemia: Results from a 24-Week Treatment Continuation Period After a 24-Week Double-Blind Placebo-Controlled Period. Calcif Tis sue Int. 2019;105(3):271-84

29. Cheong HI, Yoo H-W, Adachi M, Tanaka H, Fujiwara I, Hasegawa Y, et al. First-in-Asian Phase I Study of the Anti-Fibroblast Growth Factor 23 Monoclonal Antibody, Burosumab: Safety and Pharmacodynamics in Adults With X-linked Hypophosphatemia. JBMR Plus. 2019;3(2):e10074.

30. Lyseng-Williamson KA. Burosumab in X-linked hypophosphatemia: a profile of its use in the USA. Drugs Ther Perspect Ration Drug Sel Use. 2018;34(11):497-506.

31. Clinical manifestations, diagnosis, and treatment of osteomalacia [Internet]. Base de datos UptoDate: Peter J Snyder; 2017 [modificado en Abril 2018; citado Mayo 2018]. Disponible en: https://bit.ly/2roolXT 